\title{
The PoGOLite balloon-borne soft gamma-ray polarimeter
}

\author{
M. Kiss* \\ for the PoGOLite collaboration \\ Particle and Astroparticle Physics, Royal Institute of Technology, \\ SE-106 91 Stockholm, Sweden \\ *E-mail: mozsi@kth.se
}

PoGOLite is a balloon-borne experiment that will measure the polarization of soft gamma-rays between $25 \mathrm{keV}$ and $80 \mathrm{keV}$ through detection of coincident Compton scattering and absorption in a close-packed array of 217 well-type phoswich detector cells. The potential observation targets include pulsars, accreting compact objects and astrophysical jets. The polarization properties of such radiation can reveal important new information on the geometry, magnetic fields and emission mechanisms of these sources.

Keywords: Polarimetry, X-rays, Gamma-rays, Well-type phoswich detectors

\section{Introduction}

$\mathrm{X}$-ray and gamma-ray polarimetry is expected to provide important new information about astronomical sources, e.g. on the physical state and geometry of compact objects and the intervening material. Although potential targets for polarimetric measurements are numerous, only one significant detection of polarization in the X-ray band has been reported so far, from an experiment where the polarization of $2.6 \mathrm{keV}$ and $5.2 \mathrm{keV}$ photons from the Crab nebula was studied $[1,2]$. One of the biggest difficulties with polarimetry in the X-ray band is the high levels of background, e.g. atmospheric gamma-rays and neutrons, charged cosmic rays and neutrons produced in cosmic ray interactions with the payload mechanics. We are therefore constructing a polarimeter based on phoswich detector cell technology and several active and passive systems for background rejection. This balloon-borne instrument, PoGOLite (the Polarized Gamma-ray Observer - light-weight version) [3], operates in the energy range $25 \mathrm{keV}$ to $80 \mathrm{keV}$ and will be able to measure a $10 \%$ polarization from a $200 \mathrm{mCrab}$ source in a single six hour at an altitude of about $40 \mathrm{~km}$. Sources of interest include pulsars, accreting black holes and neutron stars, as well as galactic 
binaries and jets from active galaxies. Since photons have a higher probability to scatter perpendicular than parallel to the polarization vector [4], the distribution of azimuthal scattering angles observed in a segmented detector is modulated by the polarization, thus enabling the polarization characteristics (degree and angle) to be reconstructed [5]. The PoGOLite instrument (Fig. 1) employs a close-packed array of 217 well-type phoswich detector cells (PDCs) to detect Compton scattering and photoabsorption in order to reconstruct azimuthal scattering angles of incident photons. A 54segment side anticoincidence shield (SAS) made of BGO crystals surrounds the array and is used to detect charged particles and photons entering the instrument from the side. The neutron-induced background is reduced by a $10 \mathrm{~cm}$ thick polyethylene shield, which covers the side and the bottom of the detector. To minimize systematical bias, the instrument is also rotated around the viewing axis.

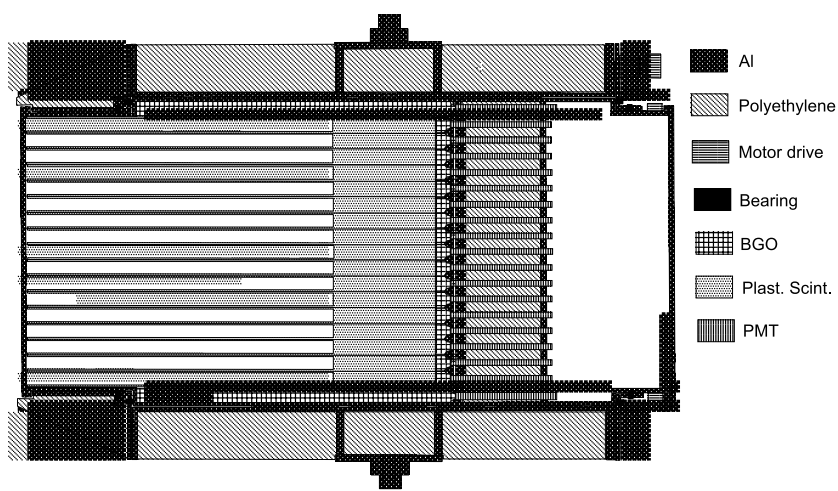

Fig. 1. Axial cross-section of the PoGOLite instrument, which measures about $1.5 \mathrm{~m}$ in length. The inner cylinder houses the active detector components, i.e. the phoswich detector cells and the side anticoincidence shield. The outer cylinder contains the polyethylene neutron shield and provides an interface for the mechanisms which enable the instrument to rotate around the viewing axis as well as pivot for elevation pointing.

Each PDC $[6,7]$ in the array consists of a hollow slow plastic scintillator $(60 \mathrm{~cm})$, a solid fast plastic scintillator $(20 \mathrm{~cm})$ and a BGO crystal $(4 \mathrm{~cm})$ and is viewed by a single photomultiplier tube. The slow scintillator is an active collimator which narrows the field of view to about 5 square degrees. Photons from the observed source enter cleanly through the hollow slow scintillator and undergo Compton scattering or photoelectric absorption in the solid fast scintillator, which is the active detector component. The bottom BGO crystal is part of the anticoincidence system and identifies back- 
entering charged particles and photons. Pulse shape discrimination is used to distinguish between signals in the different detector components [6].By measuring a Compton scattering in one cell and a photoelectric absorption in a separate cell, the azimuthal scattering angle of the incident photons can be reconstructed. The relative energy deposition is used to distinguish between scattering sites and absorption sites. Multiple-site events triggering more than two cells can also occur. Monte Carlo simulations based on the Crab pulsar spectrum show, however, that the polarization can be reliably reconstructed by including up to three-site events, and that less than $20 \%$ of the triggered events involve more than three cells $[8,9]$.

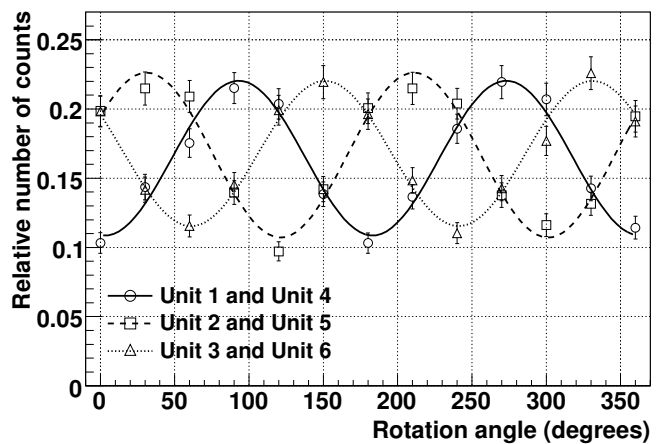

Fig. 2. Observed modulation for the $50 \mathrm{keV}$ measurement. Units 1-6 are symmetrically placed around a central unit, which is directly irradiated and acts as a scatterer. As the detector array is rotated relative to the fixed beam polarization, a modulation is seen in the relative number of counts in the six surrounding units. The modulation is more pronounced the higher the polarization degree of the incident beam is. Results shown here have been pairwise averaged for opposing detector cells to account for intrinsic differences between the cells. Sinusoidal curves have been fitted to the data.

\section{The PoGOLite prototype}

In March 2007, a 7-unit prototype was tested at the KEK "Photon Factory" in Japan [10]. The instrument was irradiated with a beam of photons with a polarization degree of $(91 \pm 1) \%$. Using flight-version front-end electronics, the scattering angles were measured at $25 \mathrm{keV}, 50 \mathrm{keV}$ and $70 \mathrm{keV}$. The detector array was rotated $360^{\circ}$ around the beam axis in $15^{\circ}$ steps while the polarization of the incident photon beam was kept constant. In-flight, the detector will be rotated in a similar fashion. Figure 2 shows the modulation obtained for the $50 \mathrm{keV}$ measurement. The modulation factor (amplitude 
of the fitted modulation curve divided by the average value of the curve) was found to be $(33.7 \pm 1.3) \%$, in accordance with our expectations. To test the background rejection capability, the detector array was irradiated by $662 \mathrm{keV}$ photons from ${ }^{137} \mathrm{Cs}$ through a segment of the side anticoincidence BGO shield. The modulation caused by the polarization could still be clearly measured, even with this added source of background. In this case, the obtained modulation factor was $(31.8 \pm 0.7) \%$.

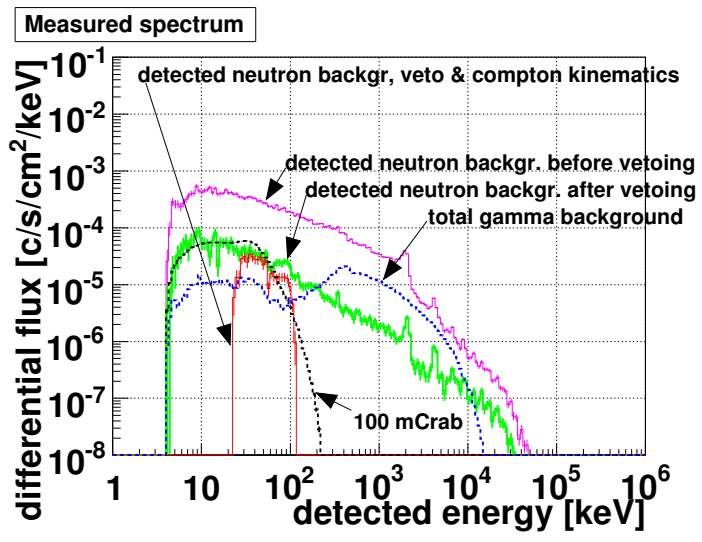

Fig. 3. Simulations of atmospheric and structure-induced contributions to the background. The energy range relevant for PoGOLite is $25 \mathrm{keV}$ to $80 \mathrm{keV}$.

\section{Background suppression}

Sources of background have been carefully studied using Geant 4 simulations $[8,11]$. Several measurements have also been made with charged particles such as $392 \mathrm{MeV}$ protons from a beam line irradiating the fast scintillator [12] or minimum ionizing electrons with energies up to $2 \mathrm{MeV}$ from ${ }^{90} \mathrm{Sr}$ irradiating the slow scintillator of a single detector cell [9]. A signal from $59.5 \mathrm{keV}$ photons from ${ }^{241} \mathrm{Am}$ could be seen even with a background greater than that expected in-flight by more than an order of magnitude. Simulations [11] show that the biggest contribution to the background is from atmospheric neutrons. The side and the bottom of the PoGOLite instrument are therefore surrounded by a polyethylene shield which either prevents the neutrons from entering the detector array at all or moderates the neutrons to energies where the probability for fake triggers is low. Figure 3 shows the simulated background from neutrons as measured by the 
instrument. By using the veto systems and Compton kinematics of the involved processes, the background can be reduced to less than a $100 \mathrm{mCrab}$ level in the relevant energy range, $25 \mathrm{keV}$ to $80 \mathrm{keV}$. Simulations also show that the background from neutrons produced when cosmic rays hit the gondola is much smaller than the other backgrounds.

\section{Mission plans and outlook}

The first flights $(2009,2010)$ will focus on the Crab pulsar, the main northern hemisphere target, and will enable the correct high-energy emission model to be identified from the three currently proposed models [3]. Other targets of interest on the northern hemisphere include Cygnus X-1, which is an X-ray binary system [13], and Hercules X-1, an accretion-powered pulsar [14]. Polarimetric measurements of Cygnus X-1 with PoGOLite will provide important new information about the emission mechanism and the accretion geometry of the binary system, whereas for the case of Hercules X-1, the measurements will give insight into the magnetic field orientation radiative processes in accretion flows onto the pulsar [3]. Long-duration flight from Sweden to Canada are also planned, during which it will be possible to observe multiple targets and study time variations of the sources. Furthermore, thanks to the simple instrumental design, PoGOLite can quickly be brought from stand-by to full operation. Future observations may therefore also focus on "targets of opportunity" such as transient events detected by GLAST, SWIFT or other instruments.

\section{References}

1. M. C. Weisskopf, et al., ApJ, 208 (1976) L125.

2. M. C. Weisskopf, et al., ApJ, 220 (1978) L117.

3. T. Kamae, et al., arXiv:0709.1278v1 [astro-ph].

4. T. Mizuno, et al., Nucl. Instr. and Meth. A 540 (2005) 158.

5. F. Lei, et al., Space Sci. Rev. Vol. 82 (1997) 309.

6. Y. Kanai, et al., Nucl. Instr. and Meth. A 570 (2007) 61-71.

7. M. Kiss, KTH Master's Thesis (2006), www.particle.kth.se/pogolite

8. O. Engdegård, KTH Master's Thesis (2006), www.particle.kth.se/pogolite

9. M. Kiss, M. Pearce, Nucl. Instr. and Meth. A 580 (2007) 876-879.

10. M. Ueno, et al., manuscript in preparation for publication

11. J. Kazejev, KTH Master's Thesis (2007), www.particle.kth.se/pogolite

12. Y. Kanai, Tokyo Institute of Technology Master's thesis (2007), kipac.stanford.edu/pogolite/collab

13. D. R. Gies, C. T. Bolton, ApJ, 304, 371 (1986).

14. P. Mészáros, et al., ApJ, 324, 1056 (1988). 